\title{
Effect of Application and Intensity of Bevacizumab-based Maintenance After Induction Chemotherapy With Bevacizumab for Metastatic Colorectal Cancer: A Meta-analysis
}

\author{
Alexander Stein, ${ }^{1}$ Carsten Schwenke, ${ }^{2}$ Gunnar Folprecht, ${ }^{3}$ Dirk Arnold ${ }^{4,5}$
}

\begin{abstract}
The role of bevacizumab-based maintenance after first-line bevacizumab-based induction treatment of metastatic colorectal cancer was evaluated in a meta-analysis of 3 randomized trials. Maintenance treatment with bevacizumab with or without fluoropyrimidines improved progression-free survival and showed a trend toward improved overall survival.

Background: The administration and intensity of bevacizumab-based maintenance therapy after induction treatment with bevacizumab is still a matter of debate. Thus, the present meta-analysis and an indirect comparison were performed to clarify these issues. Patients and Methods: Trials evaluating a separately defined "maintenance phase," with randomization after the induction phase, were selected. Three trials of maintenance with bevacizumab with or without a fluoropyrimidine (CAIRO3, SAKK 41/06, and AIO KRK 0207) were analyzed regarding the effect on progression-free survival (PFS) and overall survival (OS) of any maintenance therapy compared with observation alone and different maintenance intensities (bevacizumab with or without fluoropyrimidine) compared with observation alone and between each other. Results: Maintenance with bevacizumab with or without fluoropyrimidine after bevacizumabbased induction treatment for 4 to 6 months significantly improved PFS (hazard ratio [HR], 0.57; 95\% confidence interval $[\mathrm{Cl}], 0.43-0.75 ; P=.0004)$ and showed a trend toward prolonged OS $(\mathrm{HR}, 0.89 ; 95 \% \mathrm{Cl}, 0.78-1.02 ; P=.09)$ compared with observation alone. The effect on PFS increased with the intensity of the maintenance regimen (HR, $0.72 ; 95 \% \mathrm{Cl}, 0.60-0.85$ for single-agent bevacizumab vs. $\mathrm{HR}, 0.45 ; 95 \%, \mathrm{Cl} 0.39-0.51$ for combination therapy, both compared to observation alone). In contrast, the HRs for OS remained in the same range. A similarly improved PFS ( $\mathrm{HR}, 0.63 ; 95 \% \mathrm{Cl}, 0.50-0.79$ ) was shown for the more intensive maintenance therapy (bevacizumab and fluoropyrimidine) compared with bevacizumab alone. Conclusion: Bevacizumab-based maintenance therapy after induction chemotherapy with bevacizumab significantly improves PFS and showed a trend toward prolonged OS and should thus be considered, in particular, in patients with a response to induction treatment.
\end{abstract}

Clinical Colorectal Cancer, Vol. 15, No. 2, e29-39 @ 2015 Elsevier Inc. All rights reserved. Keywords: Bevacizumab, Fluoropyrimidines, Maintenance, Metastatic Colorectal Cancer

\section{Introduction}

Colorectal cancer (CRC) is one of the most commonly diagnosed malignancies and one of the leading causes of cancer death. ${ }^{1}$

Presented in part at the American Society of Clinical Oncology Annual Meeting, Chicago, 2014.

${ }^{1}$ Hubertus Wald Tumour Centre, University Cancer Centre Hamburg; and Second Department of Internal Medicine, University Medical Centre Hamburg-Eppendorf, Hamburg, Germany

${ }^{2}$ Schwenke Consulting: Strategies and Solutions in Statistics, Berlin, Germany

${ }^{3}$ Medical Department I, University Hospital Carl Gustav Carus, University Cancer

Center, Dresden, Germany

${ }^{4}$ CUF Hospitals Cancer Centre, Lisbon, Portugal
Approximately one quarter of patients with CRC will present with metastatic disease (mCRC) at diagnosis (synchronous disease), and about $40 \%$ of patients will develop metachronous metastases

${ }^{5}$ Hubertus Wald Tumour Centre, University Cancer Centre Hamburg, University Medical Centre Hamburg-Eppendorf, Hamburg, Germany

Submitted: Jul 16, 2015; Revised: Dec 4, 2015; Accepted: Dec 9, 2015; Epub: Dec 18, 2015

Address for correspondence: Alexander Stein, MD, Hubertus Wald Tumor Centre, University Cancer Centre Hamburg, Department of Oncology, Hematology, BMT with Section Pneumology, University Medical Centre Hamburg-Eppendorf, Martinistrasse 52, Hamburg 20246, Germany

E-mail contact: a.stein@uke.de 


\section{Effect of Application and Intensity of Bevacizumab-based Maintenance Therapy}

after curative intent treatment, resulting in the relatively high overall mortality rate associated with CRC. ${ }^{2}$

Several drugs as single agents or in various combinations are available to treat mCRC, including fluoropyrimidines (5fluorouracil [5-FU], capecitabine), irinotecan, oxaliplatin, the vascular endothelial growth factor (VEGF) antibody bevacizumab, the epidermal growth factor receptor (EGFR) antibodies cetuximab and panitumumab for $R A S$ wild-type $\mathrm{mCRC}$, the VEGF receptors 1 and 2 fusion protein aflibercept, and the multitarget tyrosine kinase inhibitor regorafenib. The current standard first-line treatment is a combination of a chemotherapy doublet of a fluoropyrimidine with oxaliplatin and/or irinotecan (FOLFOX [folinic acid, 5-FU, oxaliplatin], CAPOX [capecitabine, oxaliplatin], FOLFIRI [folinic acid, 5-FU, irinotecan] regimens) plus bevacizumab or an EGFR antibody (cetuximab or panitumumab) for patients with $R A S$ wild-type tumors. As a result of recent advances in treatment, median overall survival (OS) can now be as long as 30 months in selected patient groups. $^{3-6}$

The first-line treatment will be the longest treatment phase and is thus of particular importance regarding the overall treatment strategy. The established standard for many years was continuation of the initial treatment until the occurrence of progression or unacceptable toxicity. Newer strategies, developed particularly for oxaliplatin-based regimen, have used a limited concept of "induction and maintenance," with several strategies used in addition to the previous standard of continuation of full treatment until progression (Table 1). These strategies were initially triggered by the dose-cumulative neurotoxicity associated with oxaliplatin. In addition to the sparing of cumulative side effects (and thus improving patient quality of life), continuation of induction treatment beyond 3 to 6 months in a palliative situation without a chance for secondary local ablation does not seem to be beneficial. Therefore, induction/maintenance strategies to date have used a 3- to 6-month duration of induction chemotherapy, including other regimen than only FOLFOX. Although intermittent treatment with preplanned maintenance or full-stop intervals was evaluated in several trials, this approach has not been widely adopted. ${ }^{7-9}$ Recent trials allocated

\begin{tabular}{|c|c|c|}
\hline le 1 & $\begin{array}{l}\text { Currently Available Maintenance } \\
\text { Respective Randomized Trials }\end{array}$ & d \\
\hline \multicolumn{2}{|c|}{ Strategy } & Trials \\
\hline \multicolumn{3}{|c|}{ Partial stop and go with maintenance } \\
\hline \multicolumn{2}{|c|}{$\begin{array}{l}\text { Stop most toxic drug (eg, oxaliplatin), apply } \\
\text { maintenance (single-agent } 5 \text {-fluorouracil, } \\
\text { bevacizumab, or cetuximab or } 5 \text {-fluorouracil plus } \\
\text { bevacizumab); if feasible, restart induction } \\
\text { treatment at progression }\end{array}$} & $\begin{array}{l}\text { OPTIMOX 2, COIN-B, } \\
\text { CAIRO3, SAKK 41/06, } \\
\text { MACRO, Turkish Oncology } \\
\text { Group - Stop and } \\
\text { Go - Trial, AIO KRK } 0207\end{array}$ \\
\hline \multicolumn{2}{|c|}{$\begin{array}{l}\text { Stop/restart toxic drug (eg, oxaliplatin) in } \\
\text { preplanned intervals }\end{array}$} & OPTIMOX 1, CONcePT \\
\hline \multicolumn{3}{|c|}{ Complete stop and go } \\
\hline \multicolumn{2}{|c|}{$\begin{array}{l}\text { Stop all drugs after } 3 \mathrm{mo} \text {, restart at progression, } \\
\text { and stop again after another } 3 \text { mo }\end{array}$} & COIN \\
\hline \multicolumn{2}{|c|}{ Stop/restart all drugs in preplanned intervals } & GISCAD \\
\hline \multicolumn{3}{|c|}{ Switch maintenance } \\
\hline \multicolumn{2}{|c|}{$\begin{array}{l}\text { Introduce new drug into maintenance strategy } \\
\text { (adding erlotinib to bevacizumab maintenance) }\end{array}$} & DREAM, Nordic ACT \\
\hline
\end{tabular}

patients after 3 to 6 months of induction treatment to different maintenance strategies and/or observation alone. ${ }^{10-16}$ These trials all showed an effect on progression-free survival (PFS) or the respective alternate endpoints eg, time to failure of strategy or time to second progression; however, the effect on OS has remained unclear. The current meta-analysis was performed to compare observation alone with bevacizumab or bevacizumab and chemotherapy maintenance after induction chemotherapy in terms of PFS and OS.

\section{Patients and Methods \\ Definition of Analysis and Trial Selection}

Pertinent data from published trials (PubMed) and abstracts presented at selected oncology association meetings (American Society of Clinical Oncology and European Society for Medical Oncology/European Cancer Organization) until June 2015 were systematically searched using $\mathrm{MeSH}$ and full-text search terms for "metastatic colorectal cancer," "first-line treatment," or "maintenance treatment." In addition, the references of all eligible studies were manually reviewed to find additional relevant studies.

Overall, 9 trials were identified that had compared any maintenance strategy until progression with observation alone or the continuation of full treatment (Figure 1; Table 2). ${ }^{10-12,14,15,17-19}$ Of these, 5 trials were selected for additional analysis, because these trials evaluated a separately defined "maintenance phase," with randomization after the induction phase (OPTIMOX 3/DREAM [Optimized Leucovorin Fluorouracil Oxaliplatin/Double Inhibition Reintroduction Erlotinib Avastin Metastatic Colorectal Cancer], Nordic ACT [Chemotherapy and Avastin Followed by Maintenance Treatment With Avastin +/- Tarceva], CAIRO 3 [CApecitabine, IRinotecan and Oxaliplatin], SAKK 41/07 [Swiss Group for Clinical Cancer Research], AIO KRK 0207 [Workinggroup Medical Oncology Colorectal Cancer]). The selected studies were assessed for potential bias with regard to the randomization approach, timely parallel enrollment, similarity of treatment groups, data-driven reporting, analysis population (intent-to-treat [ITT] vs. other), and other aspects. The OPTIMOX 3/DREAM and Nordic ACT trials evaluated the addition of erlotinib to bevacizumab maintenance. ${ }^{10,19}$ However, erlotinib has not been licensed for mCRC treatment, and approval, considering the data obtained, seems unlikely.

To clarify the most relevant issues to date, the 3 trials evaluating bevacizumab with or without fluoropyrimidine maintenance (CAIRO 3, SAKK 41/06, and AIO KRK 0207) were selected for the main analysis because of their similar design (comparison of bevacizumab-based maintenance with observation alone; Figure 2). ${ }^{13-15}$ The combined analysis of these data was performed to evaluate the following questions:

1. Effect of any bevacizumab-based (with or without chemotherapy) maintenance compared with observation alone

2. Effect of bevacizumab maintenance compared with observation alone

3. Effect of fluoropyrimidine and bevacizumab maintenance compared with observation alone

4. Indirect comparison of different maintenance intensities (fluoropyrimidine and bevacizumab compared with bevacizumab alone) 


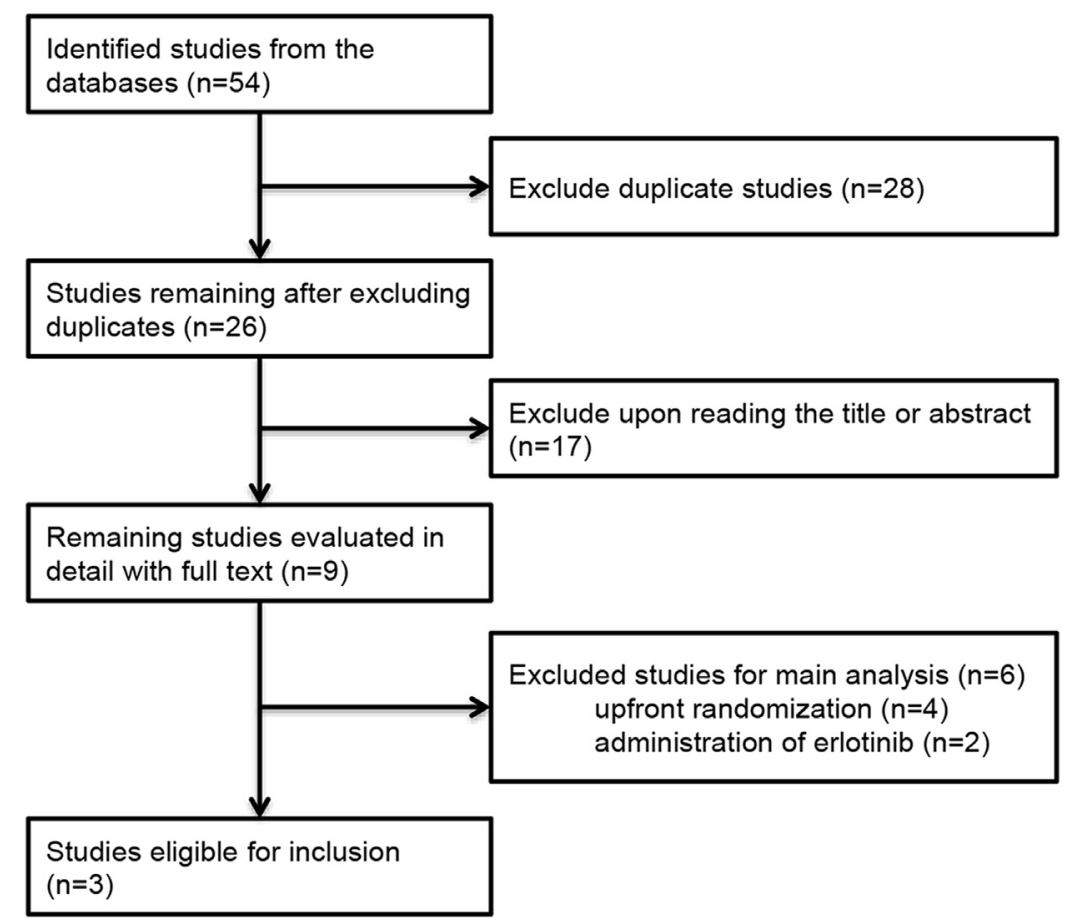

To answer the fourth question regarding the different maintenance intensities, the 2 single-agent bevacizumab arms (SAKK 41/06 and AIO KRK 0207) were compared with the 2 fluoropyrimidine and bevacizumab arms (AIO KRK 0207 and CAIRO 3). The metaanalysis of these 3 similarly performed trials was performed to combine the currently available data and provide a clinically relevant

\section{Table 2 Currently Available Randomized Clinical Trials Evaluating Different Maintenance Strategies}

\begin{tabular}{|c|c|c|c|c|c|c|}
\hline Strategy & Randomization & Clinical Trial & Induction Regimen & Maintenance Regimen & Patients (n) & Comment \\
\hline \multirow[t]{5}{*}{$\begin{array}{l}\text { Maintenance vs. } \\
\text { observation }\end{array}$} & Upfront & OPTIMOX 2 & FOLFOX & 5-FU/LV vs. observation & 202 & $\begin{array}{l}\text { Excluded owing to different } \\
\text { maintenance regimen }\end{array}$ \\
\hline & & COIN-B & FOLFOX + cetuximab & Cetuximab vs. observation & 169 & $\begin{array}{l}\text { Excluded because no } \\
\text { formal comparison }\end{array}$ \\
\hline & After induction & CAIR03 & CAPOX + bevacizumab & $\begin{array}{c}\text { Capecitabine + bevacizumab } \\
\text { vs. observation }\end{array}$ & 558 & Included \\
\hline & & SAKK 41/06 & $\begin{array}{c}\text { Chemotherapy + bevacizumab } \\
\text { (32\% 5-FU + Iri; 62\% } \\
5-\mathrm{FU}+\text { 0xa) }\end{array}$ & Bevacizumab vs. observation & 262 & Included \\
\hline & & AlO KRK 0207 & 5-FU + Oxa + bevacizumab & $\begin{array}{l}\text { Bevacizumab } \pm 5 \text {-FU/LV or } \\
\text { capecitabine vs. observation }\end{array}$ & 472 & Included \\
\hline \multirow[t]{2}{*}{$\begin{array}{l}\text { Different } \\
\text { maintenance } \\
\text { regimen }\end{array}$} & After induction & OPTMOX 3/DREAM & $\begin{array}{c}\text { Chemotherapy + bevacizumab } \\
(90 \% 5 \text {-FU + Oxa; } 10 \% \\
\text { FOLFIRI) }\end{array}$ & $\begin{array}{l}\text { Bevacizumab vs. } \\
\text { bevacizumab }+ \text { erlotinib }\end{array}$ & 452 & $\begin{array}{l}\text { Excluded owing to use of } \\
\text { bevacizumab in both arms }\end{array}$ \\
\hline & & Nordic ACT & Chemotherapy + bevacizumab & $\begin{array}{l}\text { Bevacizumab vs. } \\
\text { bevacizumab }+ \text { erlotinib }\end{array}$ & 159 & \\
\hline \multirow[t]{2}{*}{$\begin{array}{l}\text { Maintenance vs. } \\
\text { continuation of } \\
\text { full treatment }\end{array}$} & Upfront & MACRO & CAPOX + bevacizumab & $\begin{array}{c}\text { Bevacizumab vs. } \\
\text { CAPOX + bevacizumab }\end{array}$ & 470 & $\begin{array}{c}\text { Comparison of full } \\
\text { treatment continuation vs. } \\
\text { maintenance }\end{array}$ \\
\hline & & $\begin{array}{l}\text { Turkish Oncology } \\
\text { Group - Stop and } \\
\text { Go - Trial }\end{array}$ & CAPOX + bevacizumab & $\begin{array}{l}\text { Capecitabine + bevacizumab } \\
\text { vs. CAPOX + bevacizumab }\end{array}$ & 123 & \\
\hline
\end{tabular}

Abbreviations: 5-FU = 5-fluorouracil; CAPOX = capecitabine, oxaliplatin; FOLFIRI = folinic acid, 5-FU, irinotecan; FOLFOX = folinic acid, 5-FU, oxaliplatin; Iri = irinotecan; LV = leucovorin; Oxa $=$ oxaliplatin. 


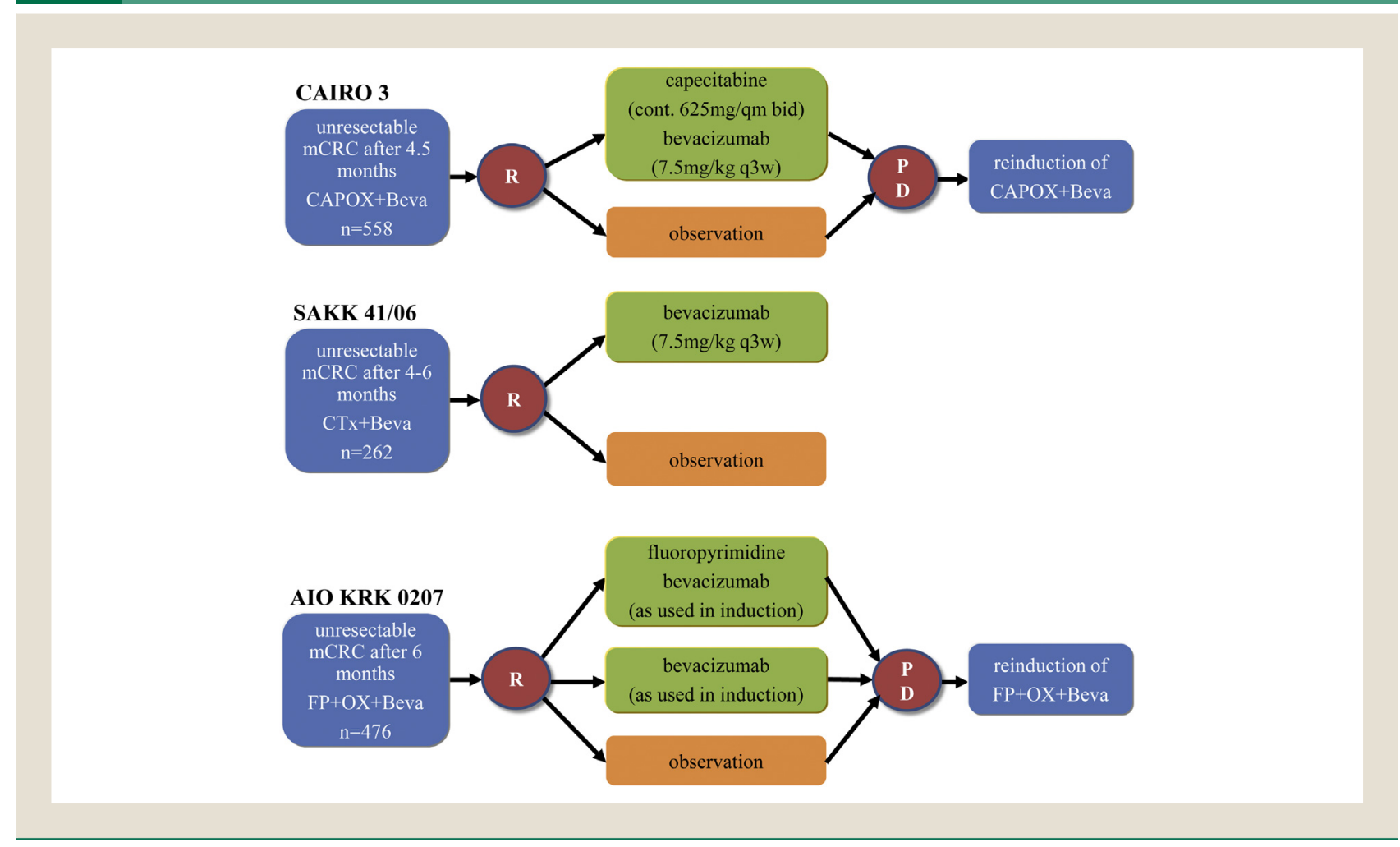

Abbreviations: Beva = bevacizumab; cont. = continuous; $\mathrm{CTx}=$ cetuximab; $\mathrm{mCRC}=$ metastatic colorectal cancer; $\mathrm{OX}=$ oxaliplatin; $\mathrm{qm}=$ every month.

conclusion regarding the use of bevacizumab-based maintenance therapy after induction treatment with bevacizumab and chemotherapy.

The 4 remaining trials had randomized patients upfront (before induction treatment) and thus could not be analyzed for the effect of different maintenance strategies, at least not regarding PFS during maintenance therapy.

\section{Statistical Analysis}

The PFS and OS were analyzed in meta-analyses and using an indirect comparison. All meta-analyses were performed using the software Review Manager, version 5.3 (The Nordic Cochrane Centre, The Cochrane Collaboration, Copenhagen, Denmark), based on the reported hazard ratios (HRs) and 95\% confidence intervals (CIs) from each study using random effects models. ${ }^{20} \mathrm{~A}$ test for heterogeneity was performed to assess for deviations from homogeneity of the studies and to assess whether the studies could be summarized in a meta-analysis. If the $P$ value for heterogeneity was $\geq .20$, no proof for heterogeneity was concluded, and a metaanalysis was performed. With proof of heterogeneity $(P<.20)$, considerable heterogeneity was concluded, and the studies were not summarized in a meta-analysis. The cause of heterogeneity was assessed, and only those studies were combined that did not show considerable heterogeneity. In addition, an indirect comparison was performed to compare combination therapy against single-agent bevacizumab using "observation" as the bridge comparator. The approach by Bucher et $\mathrm{al}^{21}$ was used to perform the comparison according to the meta-analytical results. A 95\% CI for the $\mathrm{HR}<1$ would show statistically significant results in favor of combination therapy. ${ }^{21}$ The indirect comparison was performed using Statistical Analysis Systems, version 9.2 (SAS Institute Inc., Cary, NC).

\section{Results}

The 3 studies comparing observation alone with bevacizumab with or without fluoropyrimidine after induction treatment (Figure 1), which had been selected for the meta-analyses and indirect comparison, were assessed for their potential for bias. All 3 studies were fully published randomized controlled trials with an adequate randomization approach and timely parallel enrollment. All 3 studies were open-label studies, and no crossover was allowed. The reported analyses for the endpoints of PFS and OS were standard approaches based on the ITT population; thus, data-driven reporting could be excluded. In the absence of any other aspect, the potential for bias was assessed as low for the endpoint OS in all 3 studies. Owing to the open-label design of the studies and the reading of the radiologic images at the study sites and not centrally, the endpoint PFS was suggested to have a high potential for bias. Therefore, the PFS results should be interpreted cautiously.

The studies with any active maintenance (bevacizumab with or without fluoropyrimidine), after bevacizumab-based induction treatment for 4 to 6 months, showed improved PFS (Figure 3). However, a meta-analysis showed considerable heterogeneity $(P=$ $.0004)$. Thus, the 3 studies were not combined but were separated into single-agent bevacizumab and combination bevacizumab and 


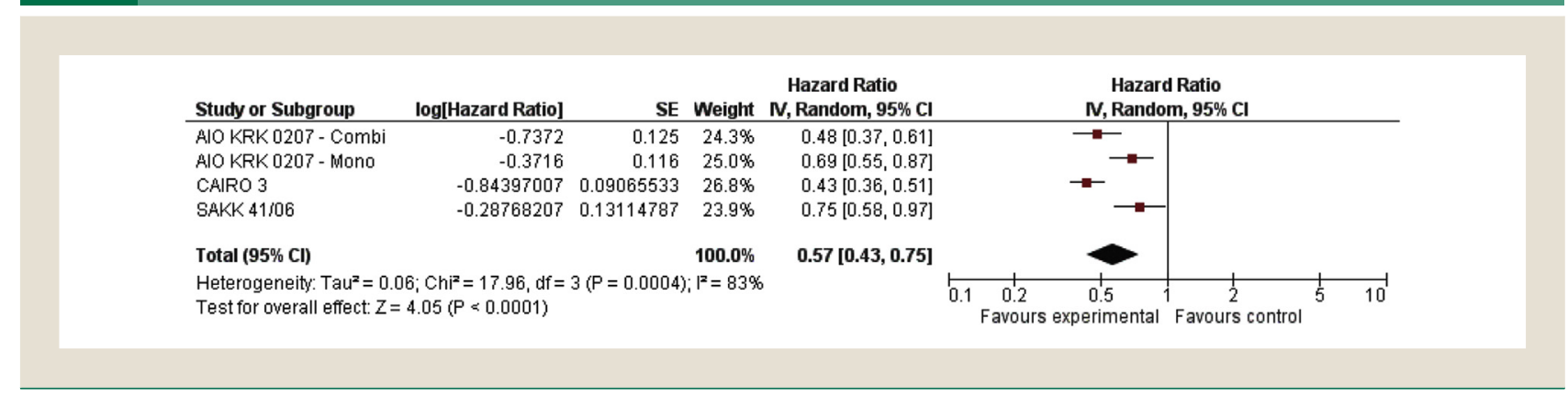

Abbreviations: $\mathrm{Cl}=$ confidence interval; Combi = combination therapy; Mono = monotherapy; $\mathrm{SE}=$ standard error.

fluoropyrimidine. In contrast, with regard to mortality, the studies were not heterogeneous $(P=.89)$. In the meta-analysis, active maintenance showed a trend toward prolonged OS (HR, 0.89; $95 \%$ CI, $0.78-1.02 ; P=.09$; Figure 4) compared with observation alone (CAIRO3, SAKK 41/06, and AIO KRK 0207). Maintenance with single-agent bevacizumab significantly increased PFS (HR, 0.72; 95\% CI, $0.60-0.85 ; P=.0001)$ and showed a trend toward prolonged OS (HR, 0.85; 95\% CI, 0.69-1.05; $P=.14$ ) compared with observation alone (see Supplemental Figures 1 and 3 in the online version). The more intensive maintenance strategy with bevacizumab and fluoropyrimidine compared with observation alone seemed to have a stronger effect on PFS (HR, 0.45; 95\% CI, 0.39$0.51 ; P<.0001)$. In contrast, the HRs for OS remained similar to single agent bevacizumab (HR, 0.92; 95\% CI, 0.77-1.09; $P=.31$; see Supplemental Figures 2 and 4 in the online version).

Finally, the indirect comparison showed improved PFS (HR, 0.63; 95\% CI, 0.50-0.79) for the more intensive maintenance regimen (bevacizumab and fluoropyrimidine) compared with bevacizumab alone. In line with the homogenous results across all therapy strategies, no effect on OS was found in favor of 1 of these 2 maintenance strategies (HR, 1.08; 95\% CI, 0.82-1.42; Figure 5).

The consistency of direct and indirect evidence was assessed using direct evidence from AIO 0207 and indirect evidence from SAKK $41 / 06$ and CAIRO3. For PFS, heterogeneity was found for the 2 treatment arms $(P=.03)$, suggesting a treatment effect with regard to the HRs for the combination and mono-strategy (HR combination, 0.48; 95\% CI, 0.37-0.61) versus (HR monotherapy, 0.69; 95\% CI, 0.55-0.87; HR combination/HR mono, 0.70; 95\% CI,
0.49-0.98). These results are in line with the advantage of the combination strategy in the indirect comparison of the data from CAIRO3 and SAKK 41/06 (HR, 0.57; 95\% CI, 0.42-0.78). For OS, no deviance from homogeneity was found between the 2 treatment arms compared with observation $(P=.62)$ within the AIO 0207 study (HR combination, 0.99; 95\% CI, 0.71-1.38; vs. HR monotherapy, 0.88 ; $95 \% \mathrm{CI}, 0.63-1.23$ ) with a ratio of the HRs of 1.13 (95\% CI, 0.70-1.80). This is in line with the findings from the indirect comparison of CAIRO3 and SAKK 41/06 (HR, 1.07; 95\% CI, 0.76-1.51). Overall, a consistency of the direct and indirect evidence can be concluded for both endpoints evaluated.

For the combined switch maintenance with erlotinib and bevacizumab, a favorable outcome in terms of PFS (HR, 0.77; 95\% CI, $0.64-0.93 ; P=.006)$ and a trend toward a prolonged OS (HR, 0.89; 95\% CI, 0.73-1.08; $P=.24$ ) was noted (see Supplemental Figures 5 and 6 in the online version).

\section{Discussion}

The results from the current meta-analysis have clearly shown significant benefits in terms of PFS and a trend toward prolonged OS in favor of a bevacizumab-based maintenance compared with observation alone after 4 to 6 months of induction treatment. Although the intensity of maintenance bevacizumab and fluoropyrimidine versus single-agent bevacizumab showed an effect on PFS, no difference in OS was noted. Similar trends were found in the randomized AIO KRK 0207 trial comparing observation with single-agent bevacizumab and bevacizumab plus fluoropyrimidine. ${ }^{15}$ Thus, both options can be considered after 4 to 6

\section{Figure 4 Overall Survival of Maintenance With Bevacizumab With or Without Fluoropyrimidine Versus Observation}

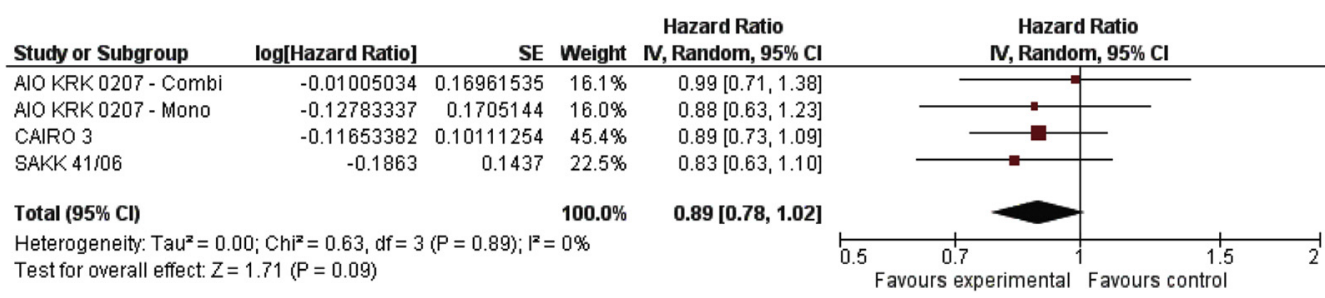

Abbreviations: $\mathrm{Cl}=$ confidence interval; Combi $=$ combination therapy; Mono = monotherapy; SE $=$ standard error. 


\section{Effect of Application and Intensity of Bevacizumab-based Maintenance Therapy}

\section{Figure 5 Indirect Comparison of Bevacizumab (BC) and Fluoropyrimidine Versus Bevacizumab}

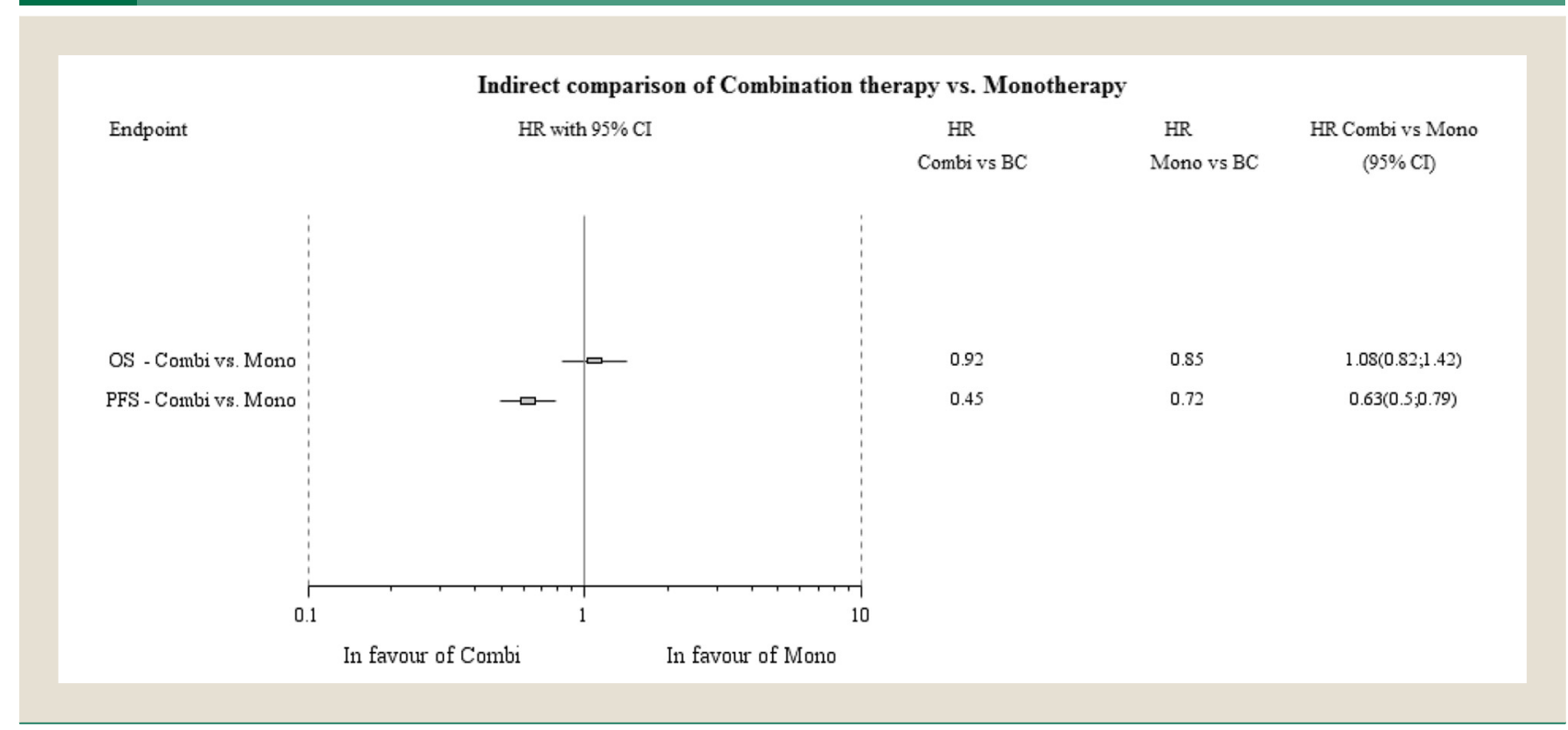

Abbreviations: $\mathrm{Cl}=$ confidence interval; Combi = combination therapy; $\mathrm{HR}=$ hazard ratio; $\mathrm{OS}=$ overall survival; PFS = progression-free survival.

months of bevacizumab-based induction treatment. Regarding the significant benefit in terms of PFS, the more intensive regimen might be chosen if durable disease control is of importance (eg, widespread and symptomatic disease at the initial presentation).

Because of the missing OS benefit, observation alone is a valid treatment approach, in particular in terms of the relevant treatment cost for bevacizumab-based maintenance therapy (estimated additional cost of approximately US\$30,000 in the SAKK 41/06 trial). Regarding the significant benefit for PFS and the noted trend for OS (HR, 0.89) with well-tolerated treatment administered for 5 to 6 months, maintenance therapy should still be considered. Both CAIRO3 and AIO KRK 0207 have consistently shown a significant benefit in terms of OS for those achieving an objective response (complete or partial response) during induction treatment. In addition, the RAS/BRAF subgroup analysis of the AIO KRK 0207 suggested that bevacizumab plus fluoropyrimidine maintenance might be better than single-agent bevacizumab in patients with mutations. Further research is needed to detect the subgroups that will benefit from maintenance therapy and to evaluate the costeffectiveness of bevacizumab-based maintenance.

Although a significantly better PFS could be shown for the combination of erlotinib and bevacizumab compared with bevacizumab alone, the relatively modest efficacy seems to be outweighed by the significant toxicity, specifically rash, diarrhea, and fatigue. ${ }^{10,19}$ Therefore, the licensing and consecutive administration of erlotinib in mCRC maintenance seems unlikely.

The present meta-analysis had several limitations, including using the published HRs rather than individual patient data. ${ }^{13-15}$ Furthermore, the heterogeneities in the trial design (superiority in CAIRO3 and noninferiority in AIO KRK 0207 and SAKK 41/06), patient inclusion criteria (eg, exclusion of patients with toxic effects from induction treatment in CAIRO3), and the treatment applied (eg, differences in induction treatment and fluoropyrimidine maintenance schedule) should be considered as potential biases. The induction treatment was heterogeneous among the trials in terms of duration, ranging from 4 to 6 months, the use of oxaliplatin $(100 \%$ of patients in CAIRO3 and AIO KRK 0207, 62\% in SAKK 41/06, with $31 \%$ receiving irinotecan), intensity ( $7 \%$ received induction treatment with fluoropyrimidine and bevacizumab in the SAKK 41/ 06 ), and the fluoropyrimidine backbone used (capecitabine/oxaliplatin at $100 \%$ in CAIRO3 and only $16 \%$ in AIO KRK 0207). The dosage of the fluoropyrimidine-based maintenance regimen differed between CAIRO3 (capecitabine with $625 \mathrm{mg} / \mathrm{m}^{2}$ twice daily continuously) and AIO KRK 0207 (capecitabine with $1000 \mathrm{mg} / \mathrm{m}^{2}$ twice daily for 2 weeks, followed by a 1 -week break, or 5-FU/leucovorin [LV] at a full dosage ranging from 2400 to $3000 \mathrm{mg} / \mathrm{m}^{2}$ continuous infusion for 2 days with or without a bolus every 2 weeks) thus potentially influencing the results of the pooled intensive maintenance arms.

Additional maintenance strategies (eg, single-agent cetuximab or 5-FU/LV compared with observation alone or bevacizumab-based maintenance compared with full treatment continuation) could not be included owing to the upfront randomization. ${ }^{11,12,17,18}$ The results from the present analysis do not allow for conclusions regarding the comparative value of bevacizumab versus fluoropyrimidines or the effect of EGFR antibodies in this setting. The discussed maintenance approach has generally been limited to an oxaliplatin-based regimen, although a small number of patients receiving an irinotecan-based induction regimen were included in the SAKK 41/06 (about 30\%) and the OPTIMOX 3/DREAM trials. In addition to the intermittent treatment approach applied in the GISCAD trial, the effect of a de-escalation strategy after an irinotecan-based first-line regimen combined with monoclonal antibodies compared with continuation of full-dose treatment remains unclear. $^{7}$

In addition, the endpoint of PFS was considered to have a high potential for bias owing to the open-label design of all 3 studies and the assessment of the radiologic images at the study sites and not 
centrally. Also, the indirect comparison had some limitations. The similarity of the studies was assessed, and no deviance was detected; however, hidden confounders could have introduced bias into the results. Owing to the limited number of studies, no sensitivity analyses were performed to assess any confounding. The high potential for bias for the PFS endpoint also led to relevant uncertainty; thus, the results should be interpreted with care. The consistency of the direct and indirect evidence was assessed using the indirect comparison and study AIO KRK 0207. No major deviances were found, suggesting a valid indirect comparison.

Regarding the remaining unresolved issues in maintenance (eg, patient or regimen selection), the evaluation of new treatment strategies or agents in the maintenance setting has gained widespread interest in the past years, with a variety of trials currently ongoing. ${ }^{22}$

\section{Conclusion}

Bevacizumab-based maintenance after induction chemotherapy with bevacizumab significantly improves PFS and shows a favorable trend in prolonging OS. Regarding the lack of a significant OS benefit, bevacizumab-based maintenance therapy after induction treatment is not the standard approach for all patients with mCRC. However, patients achieving an objective response during bevacizumab-based induction treatment seem to derive a relevant OS benefit. The intensity of the maintenance therapy (single-agent bevacizumab or combined with a fluoropyrimidine) only affects PFS. Thus, the more intensive maintenance regimens may be limited to patients in need of durable disease control or those with worse prognostic features (eg, RAS/ $B R A F$ mutation). The decision regarding maintenance therapy warrants consideration in a shared decision-making process with the patient considering disease-specific factors (eg, the response to induction treatment).

\section{Clinical Practice Points}

- Regarding the recently achieved long-term survival of patients with mCRC, continuation of full-dose systemic treatment throughout the disease course is no longer feasible.

- Different treatment approaches are available after 3 to 6 months of induction treatment (treatment break, intermittent treatment, or maintenance).

- Recent data have shown the beneficial effect of bevacizumab-based maintenance treatment compared with observation alone in terms of PFS, although the effect on OS is unclear and the intensity of maintenance treatment remains a matter of debate.

- Bevacizumab-based maintenance therapy after 4 to 6 months of bevacizumab-based induction treatment significantly improved PFS (HR, 0.57; 95\% CI, 0.43-0.75; P=.0004) and showed a trend toward prolonged OS (HR, 0.89; 95\% CI, 0.78-1.02; $P=.09)$.

- Improved PFS (HR, 0.63; 95\% CI, 0.50-0.79) was shown for the more intensive maintenance regimen (bevacizumab and fluoropyrimidine) compared with bevacizumab alone, although OS remained unaffected (HR, 1.08; 95\% CI, 0.82-1.42).

- Because of the limited effect on OS, bevacizumab-based maintenance therapy is not the standard treatment approach for all patients but should be considered for patients with a response to induction or requiring durable disease control (significant PFS benefit).

- The addition of fluoropyrimidine to bevacizumab had no effect on OS; thus, both strategies can be considered if active maintenance therapy is chosen.

\section{Acknowledgments}

The statistical analysis was supported by Roche.

\section{Disclosure}

A.S. and D.A. have received honoraria and research support from Roche; G.F. has received honoraria from Roche; and C.S. is consultant for statistics to Roche on an honorary basis.

\section{Supplemental Data}

The supplemental figures accompanying this article can be found in the online version at http://dx.doi.org/10.1016/j.clcc.2015.12.005.

\section{References}

1. Ferlay J, Soerjomataram II, Dikshit R, et al. Cancer incidence and mortality worldwide: sources, methods and major patterns in GLOBOCAN 2012. Int Cancer 2015; 136:E359-86.

2. Siegel R, Desantis C, Jemal A. Colorectal cancer statistics, 2014. CA Cancer J Clin 2014; 64:104-17.

3. Douillard JY, Oliner KS, Siena S, et al. Panitumumab-FOLFOX4 treatment and RAS mutations in colorectal cancer. N Engl J Med 2013; 369:1023-34.

4. Venook A, Niedzwiecki D, Lenz HJ, Innocenti F, Mahoney M. CALGB/SWOG 80405: phase III trial of irinotecan/5-FU/leucovorin (FOLFIRI) or oxaliplatin/5 FU/leucovorin (mFOLFOX6) with bevacizumab (BV) or cetuximab (CET) for patients (pts) with KRAS wild-type (wt) untreated metastatic adenocarcinoma of the colon or rectum (MCRC). I Clin Oncol 2014; 32(suppl), abstract LBA3.

5. Heinemann V, von Weikersthal LF, Decker T, et al. FOLFIRI plus cetuximab versus FOLFIRI plus bevacizumab as first-line treatment for patients with metastatic colorectal cancer (FIRE-3): a randomised, open-label, phase 3 trial. Lancet Oncol 2014; 15:1065-75.

6. Loupakis F, Cremolini C, Masi G, et al. Initial therapy with FOLFOXIRI and bevacizumab for metastatic colorectal cancer. N Engl J Med 2014; 371:1609-18.

7. Labianca R, Sobrero A, Isa L, et al. Intermittent versus continuous chemotherapy in advanced colorectal cancer: a randomised "GISCAD" trial. Ann Oncol 2011; 22: 1236-42.

8. Tournigand C, Cervantes A, Figer A, et al. OPTIMOX1: a randomized study of FOLFOX4 or FOLFOX7 with oxaliplatin in a stop-and-Go fashion in advanced colorectal cancer-a GERCOR study. J Clin Oncol 2006; 24:394-400.

9. Hochster HS, Grothey A, Hart L, et al. Improved time to treatment failure with an intermittent oxaliplatin strategy: results of CONcePT. Ann Oncol 2014; 25:11728.

10. Johnsson A, Hagman H, Frodin JE, et al. A randomized phase III trial on maintenance treatment with bevacizumab alone or in combination with erlotinib after chemotherapy and bevacizumab in metastatic colorectal cancer: the Nordic ACT trial. Ann Oncol 2013; 24:2335-41.

11. Chibaudel B, Maindrault-Goebel F, Lledo G, et al. Can chemotherapy be discontinued in unresectable metastatic colorectal cancer? The GERCOR OPTIMOX2 Study. J Clin Oncol 2009; 27:5727-33.

12. Wasan H, Meade AM, Adams R, et al. Intermittent chemotherapy plus either intermittent or continuous cetuximab for first-line treatment of patients with KRAS wild-type advanced colorectal cancer (COIN-B): a randomised phase 2 trial. Lancet Oncol 2014; 15:631-9.

13. Simkens LH, van Tinteren H, May A, et al. Maintenance treatment with capecitabine and bevacizumab in metastatic colorectal cancer (CAIRO3): a phase 3 randomised controlled trial of the Dutch Colorectal Cancer Group. Lancet 2015; 385:1843-52.

14. Koeberle D, Betticher DC, von Moos R, et al. Bevacizumab continuation versus no continuation after first-line chemotherapy plus bevacizumab in patients with metastatic colorectal cancer: a randomized phase III non-inferiority trial (SAKK 41/06). Ann Oncol 2015; 26:709-14.

15. Hegewisch-Becker S, Graeven U, Killing B, Depenbusch R, Steffens CC. Maintenance strategies after initial first-line oxaliplatin/fluoropyrimidine/bevacizumab for patients with metastatic colorectal cancer: AIO 0207, a randomized, open-label, phase 3 trial. Lancet Oncol 2015; 16:1355-69.

16. Tournigand C, Chibaudel B, Samson B, et al. Bevacizumab with or without erlotinib as maintenance therapy in patients with metastatic colorectal cancer 


\section{Effect of Application and Intensity of Bevacizumab-based Maintenance Therapy}

(GERCOR DREAM; OPTIMOX3): a randomised, open-label, phase 3 trial, Lancet Oncol 2015; 16:1493-505.

17. Yalcin S, Uslu R, Dane F, et al. Bevacizumab + capecitabine as maintenance therapy after initial bevacizumab + XELOX treatment in previously untreated patients with metastatic colorectal cancer: phase III "Stop and Go" study results—a Turkish Oncology Group Trial. Oncology 2013; 85:328-35.

18. Diaz-Rubio E, Gomez-Espana A, Massuti B, et al. First-line XELOX plus bevacizumab followed by XELOX plus bevacizumab or single-agent bevacizumab as maintenance therapy in patients with metastatic colorectal cancer: the phase III MACRO TTD study. Oncologist 2012; 17:15-25.

19. Tournigand C, Samson B, Scheithauer W. Bevacizumab (Bev) with or without erlotinib as maintenance therapy, following induction first-line chemotherapy plus
Bev, in patients with metastatic colorectal cancer (mCRC): efficacy and safety results of the international GERCOR DREAM phase III trial. J Clin Oncol 2012; 30 (suppl), abstract LBA3500.

20. The Nordic Cochrane Centre, The Cochrane Collaboration, RevMan version 5. 1, User Guide-Review Manager (RevMan) [Computer program]. 2011; version 5.1 .

21. Bucher HC, Guyatt GH, Griffith LE, Walter SD. The results of direct and indirect treatment comparisons in meta-analysis of randomized controlled trials. J Clin Epidemiol 1997; 50:683-91.

22. Aprile G, Giuliani F, Lutrino SE, et al. Maintenance therapy in colorectal cancer: moving the artillery down while keeping an eye on the enemy [e-pub ahead of print]. Clin Colorectal Cancer 2015. http://dx.doi.org/10.1016/j.clcc.2015.08.002. 


\section{Alexander Stein et al}

\section{Supplemental Figure 1 Progression-free Survival of Maintenance With Bevacizumab (Bev) Versus Observation (Obs)}

\begin{tabular}{|c|c|c|c|c|c|c|c|c|c|}
\hline Stucty or Subgroup & log[Hazard Ratio] & SE & Weight & $\begin{array}{l}\text { Hazard Ratio } \\
\text { IN, Random, } 95 \% \mathrm{Cl}\end{array}$ & & $\begin{array}{r}\text { Hazard } \\
\text { IV, Randor }\end{array}$ & $\begin{array}{l}\text { Ratio } \\
\mathrm{m}, \mathbf{9 5} \% \mathrm{Cl}\end{array}$ & & \\
\hline AIO KRK 0207 - Mono & -0.3711 & 0.1162 & $56.0 \%$ & $0.69[0.55,0.87]$ & & & & & \\
\hline SAKK 41:06 & -0.28768207 & 0.13114787 & $44.0 \%$ & $0.75[0.58,0.97]$ & & & & & \\
\hline Total $(95 \% \mathrm{Cl})$ & & & $100.0 \%$ & $0.72[0.60,0.85]$ & & & & & \\
\hline \multicolumn{4}{|c|}{$\begin{array}{l}\text { Heterogeneity: } \mathrm{Tau}^{2}=0.00 ; \mathrm{Chi}^{2}=0.23, \mathrm{df}=1(\mathrm{P}=0.63) ; \mathrm{I}^{2}=0 \% \\
\text { Test for owerall effect: } \mathrm{Z}=3.85(\mathrm{P}=0.0001)\end{array}$} & & $0.1 \quad 0.2$ & $\begin{array}{c}0.5 \\
\text { Favours Bev }\end{array}$ & $\begin{array}{c}\frac{1}{2} \\
\text { Favours Obs }\end{array}$ & 5 & $\overrightarrow{10}$ \\
\hline
\end{tabular}

Abbreviations: $\mathrm{Cl}=$ confidence interval; $\mathrm{Mono}=$ monotherapy; $\mathrm{SE}=$ standard error .

Supplemental Figure 2 Progression-free Survival of Maintenance With Bevacizumab and Fluoropyrimidine Versus Observation

\begin{tabular}{|c|c|c|c|c|c|c|c|c|c|}
\hline Study or Subgroup & log[Hazard Ratio] & SE & Weight & $\begin{array}{c}\text { Hazard Ratio } \\
\text { N, Random, } 95 \% \mathrm{CI}\end{array}$ & \multicolumn{5}{|c|}{$\begin{array}{c}\text { Hazard Ratio } \\
\text { IN, Random, } 95 \% \mathrm{Cl}\end{array}$} \\
\hline AlO KRK 0207 - Combi & -0.7381 & 0.1242 & $34.8 \%$ & $0.48[0.37,0.61]$ & & -2 & & & \\
\hline CAIRO 3 & -0.84397007 & 0.09065533 & $65.2 \%$ & $0.43[0.36,0.51]$ & & & & & \\
\hline Total $(95 \% \mathrm{Cl})$ & & & $100.0 \%$ & $0.45[0.39,0.51]$ & & & & & \\
\hline $\begin{array}{l}\text { Heterogeneity: } \operatorname{Tau}^{2}=0 \text {. } \\
\text { Test for overall effect: } Z\end{array}$ & $\begin{array}{l}0 ; \mathrm{Chi}^{2}=0.47, \mathrm{df}=1 \\
11.02(\mathrm{P}=0.00001)\end{array}$ & $(P=0.49) ; I^{2}=$ & & & $\begin{array}{cc} & 1 \\
0.1 & 0.2 \\
& \text { Favour }\end{array}$ & $\begin{array}{c}0.5 \\
\text { perimental }\end{array}$ & $\begin{array}{c}1 \\
2 \\
\text { Favours control }\end{array}$ & 5 & $\overrightarrow{10}$ \\
\hline
\end{tabular}

Abbreviations: $\mathrm{Cl}=$ confidence interval; $\mathrm{Combi}=$ combination therapy; $\mathrm{SE}=$ standard error. 


\section{Effect of Application and Intensity of Bevacizumab-based Maintenance Therapy}

\section{Supplemental Figure 3 Overall Survival of Maintenance With Bevacizumab Versus Observation}

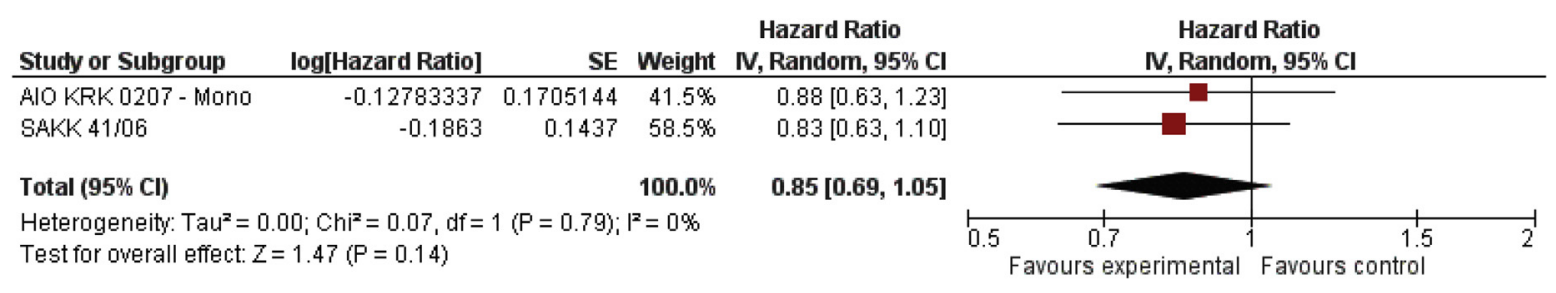

Abbreviations: $\mathrm{Cl}=$ confidence interval; Mono = monotherapy; $\mathrm{SE}=$ standard error.

\section{Supplemental Figure 4 Overall Survival of Maintenance With Bevacizumab and Fluoropyrimidine Versus Observation}

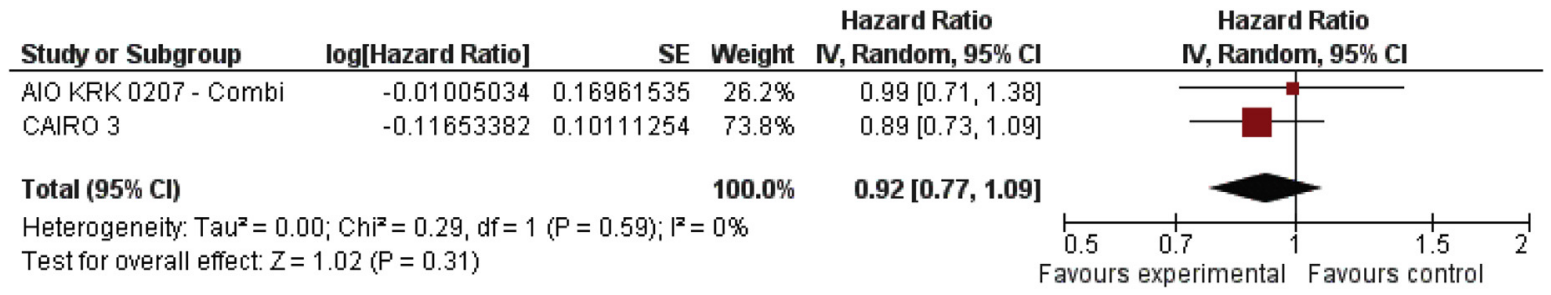

Abbreviations: $\mathrm{Cl}=$ confidence interval; $\mathrm{Combi}=$ combination therapy; $\mathrm{SE}=$ standard error. 


\section{Alexander Stein et al}

Supplemental Figure 5 Progression-free Survival of Bevacizumab (Bev) Versus Bevacizumab/Erlotinib (Bev/Erlo)

\begin{tabular}{|c|c|c|c|c|c|c|c|}
\hline Study or Subgroup & log[Hazard Ratio] & SE & Weight & $\begin{array}{c}\text { Hazard Ratio } \\
\text { IV, Random, } 95 \% \mathrm{Cl}\end{array}$ & $\begin{array}{r}\text { Hazarc } \\
\text { IV, Rando }\end{array}$ & $\begin{array}{l}\text { Ratio } \\
\mathrm{m}, \mathbf{9 5} \% \mathrm{Cl}\end{array}$ & \\
\hline Nordic ACT & -0.27443685 & 0.11217526 & $73.1 \%$ & $0.76[0.61,0.95]$ & & & \\
\hline Optimox 3 & -0.23572233 & 0.18475578 & $26.9 \%$ & $0.79[0.55,1.13]$ & & & \\
\hline Total $(95 \% \mathrm{Cl})$ & & & $100.0 \%$ & $0.77[0.64,0.93]$ & & & \\
\hline \multicolumn{5}{|c|}{$\begin{array}{l}\text { Heterogeneity: Tau }=0.00 ; \mathrm{Chi}^{2}=0.03, \mathrm{df}=1(\mathrm{P}=0.86) ;\left.\right|^{2}=0 \% \\
\text { Test for overall effect: } Z=2.75(\mathrm{P}=0.006)\end{array}$} & $\begin{array}{ll}0.5 & 0.7 \\
\text { Favours Bev/Erlo }\end{array}$ & $\begin{array}{c}1.5 \\
\text { Favours Bev }\end{array}$ & 2 \\
\hline
\end{tabular}

Abbreviations: $\mathrm{Cl}=$ confidence interval; $\mathrm{SE}=$ standard error.

Supplemental Figure 6 Overall Survival of Bevacizumab (Bev) Versus Bevacizumab/Erlotinib (Bev/Erlo)

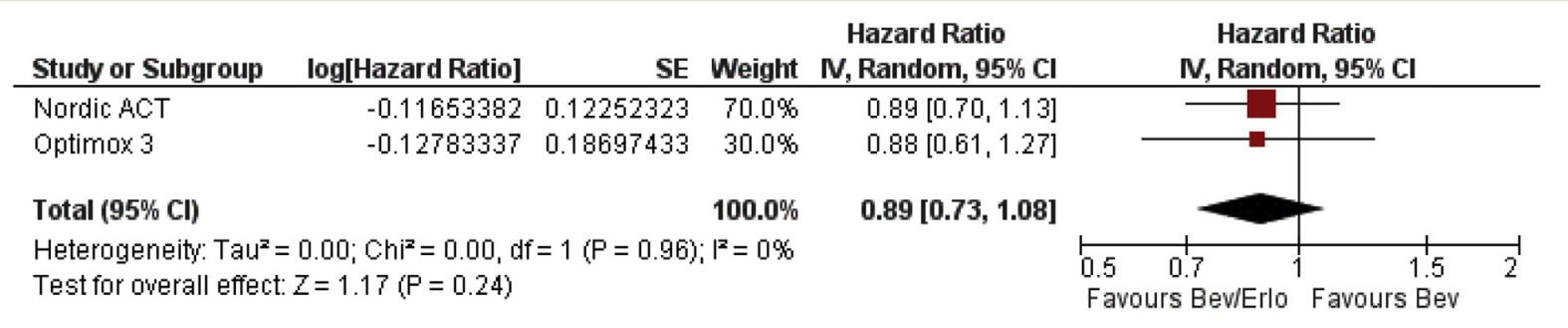

